"Biopsychosocial Aspects of Integrated Patient Care"

\title{
Use of Complementary and Alternative Medicine in patients with organ transplantation in Switzerland
}

Torchetti L, PhD, von Ammon K, MD, Frei-Erb M, MD

Institute of Complementary Medicine, University of Bern, Bern, Switzerland

Correspondence: martin.frei@ikom.unibe.ch

Introduction

The aim was to investigate retrospectively use of Complementary and Alternative Medicine (CAM) in the treatment of patients with organ transplantation in Switzerland.

Methods

Members of the Swiss transplant association completed a questionnaire about CAM use retrospectively. Five different stages were differentiated: CAM usage 1) during underlying disease, 2) before transplant, 3) during hospitalisation/rehabilitation from transplant, 4) for transplant complications and 5) after transplant for other diseases.

Results

Of the 267 patients contacted, 124 (46\%) completed the questionnaire, and data of 118 (44\%) participants could be analyzed: 55 women (47\%), mean age 56 years. Overall, 64 (54\%) indicated CAM use, with about $30 \%$ usage at every stage (except during hospitalization with only $10 \%)$.

Different methods were most common: during underlying disease classical homeopathy (15\% of all participants), before transplant dietary supplements (13\%), during hospitalization meditation (3\%), for transplantation complications dietary supplements (10\%), and after transplant for other diseases massage (11\%).

Among the 64 CAM-users, the most important reasons for the usage were improvement of general condition (36\%) and abatement of adverse effects of conventional treatment (25\%). Among the 54 non-CAM-users, most frequent reasons for not choosing CAM were insecurity about interactions with conventional treatment (46\%), and ignorance of this option (28\%). About 35\% of the CAM-users reported an improved general condition, while 30\% noticed an abatement of side effects of conventional treatment.

Conclusion

To prevent dangerous interactions with conventional treatment, more information on possibilities of CAM in the treatment of patients with transplantations is needed for doctors and patients. 